\title{
Discrimination of Ventricular Tachycardia from Ventricular Fibrillation by Morphologic Analysis of Electrograms
}

\author{
LA DiCarlo*, JM Jenkins, C Kreigler \\ * Cardiac Electrophysiology Laboratory, St. Joseph Mercy Hospital, \\ Department of Electrical Engineering and Computer Science, and School of Medicine \\ University of Michigan, Ann Arbor, MI
}

\begin{abstract}
To determine whether ventricular tachycardia (VT) and ventricular fibrillation (VF) can be discriminated by time-domain analysis, correlation waveform analysis (CWA) was performed using bipolar $(1 \mathrm{~cm}$, $1.500 \mathrm{~Hz}$ ) intracardiac electrograms of 12 consecutive patients during sinus rhythm (SR) and induced monomorphic $V T$ and VF. Using a "best fit" template-signal alignment, CWA separated $S R$ from 10/12 VTs (83\%) and 12/12 VFs (100\%), respectively. The individual variances for $V T$ and $V F$ were significantly different ( $F$ test, $p<0.0001$ ), and a threshold midway between the average variance in $V T$ and $V F$ was a successful discriminant in $11 / 12$ cases (92\%). These results suggest that time-domain analysis can discriminate $V T$ and $V F$ from $S R$, and the magnitude of the variance in $V T$ and $V F$ can be used to distinguish $V T$ from $V F$.
\end{abstract}

\section{Introduction}

Pacing-cardioverter-defibrillators are capable of providing "ramp" therapy including antitachycardia pacing, low- and high-energy cardioversion, or defibrillation for sustained ventricular tachycardia (VT) and ventricular fibrillation (VF). Appropriate therapy, however, is dependent upon correct identification of VT and VF.

Currently available, implantable devices utilize a combination of rate and a variation of amplitude distribution analyais (probability density function) to distinguish VF from SR. It has been demonstrated, however, that this algorithm is not capable of reliably detecting VT.

Morphologic analysis of intracardiac electrograms using correlation waveform analysis (CWA) has been demonstrated to be effective in discriminating normal sinus thythm (SR) from VT, paroxysmal bundle branch block of supraventricular origin, and retrograde atrial activation $[1,2,3,4]$. Whether VF can be distinguished from SR and VT with a similar timedomain analysis has not been determined.

\section{Methods}

Twelve consecutive patients were evaluated while undergoing routine cardiac electrophysiology studies. Distal bipolar (1-500 Hz) intraventricular electrograms were recorded from an electrode catheter positioned in the right ventricular apex during SR, monomorphic VT induced by programmed electrical stimulation, and VF induced by programmed stimulation or alternating current.

Ventricular electrogram, were recorded on FM magnetic tape and digitized subsequently on a PC at a sampling rate of $1,000 \mathrm{~Hz}$. The programs for digitizing and waveform analysis were written in $\mathrm{C}$ and aseembly language.

A digital differentiator was used to detect each of the individual intracardiac electrograms. A template was created from a 15 \& pacsage of SR for CWA of subeequent $15-30$ s passages of SR, VT, and VF. A patient- specific window was chosen during creation of the sinus rhythm template to include depolarization only and to exclude the potentially confounding repolarision injury current caused by the acute placement of the electrode catheters. This window size was imposed on all subeequently analyzed electrograms.

The template was aligned with the trigger point of the electrogram under analyais, and then shifted a maximum of $10 \mathrm{~ms}$ in both directions in order to maximize the alignment of the two waveforms. The magnitude of the variance of cycle-to-cycle CWA was then used as a discriminant function for VT and VF. 


\section{Experimental Results}

Table 1 summarizes the reaults for all 12 patients. The types of electrograms analyzed are illustrated in Figures 1 and 2.

The window sizes of the analyzed electrograms ranged from 52-106 ms. Mean correlation coefficient separated SR from VT in 10/12 patients (83\%) and SR from VF in all 12 patients (100\%). The range (and means) for standard deviation (s) were $\mathbf{0 . 0 0 4}$ $0.049(0.013)$ for SR, 0.007-0.177 (0.059) for VT, and $0.092-0.718(0.414)$ for VF.

For each of the 12 patients, the individual variances for VT and VF were significantly different (F test, $p$ $<0.0001)$. A threshold midway between the average variance in VT and VF, respectively, was a successful discriminant in $11 / 12$ patients (92\%).

\begin{tabular}{||c||c|c||c|c||c|c||}
\hline \hline \multicolumn{1}{|c||}{} & \multicolumn{2}{c|}{ SR } & \multicolumn{2}{c||}{ VT } & \multicolumn{2}{c||}{ VF } \\
mean & sd & mean & sd & mean & sd \\
\hline 1 & .986 & .008 & .852 & .115 & .714 & .221 \\
2 & .987 & .006 & .836 & .072 & .355 & .348 \\
3 & .995 & .003 &. .108 & .097 & .499 & .382 \\
4 & .970 & .003 & .848 & .017 & .405 & .453 \\
5 & .980 & .006 & .882 & .041 & .429 & .493 \\
6 & .962 & .020 & .641 & .047 & .625 & .408 \\
7 & .995 & .002 & .925 & .007 & .650 & .433 \\
8 & .990 & .021 & .969 & .013 & .347 & .432 \\
9 & .984 & .011 & .868 & .022 & .228 & .490 \\
10 & .977 & .049 & .960 & .018 & .881 & .092 \\
11 & .959 & .024 & .040 & .177 & .162 & .499 \\
12 & .996 & .003 & .870 & .087 & .313 & .718 \\
\hline \hline
\end{tabular}

Table 1: Results of Correlation Waveform Analysis for Discriminating Ventricular Tachycardia from Ventricular Fibrillation.

\section{Discussion}

The efficacy of implantable electrical devices such as pacing-cardioverter- defibrillators is dependent upon accurate identification of rapid ventricular tachyarrhythmias such an monomorphic VT and VF. The primary method for tachycardis detection by implantable devices bas been baced upon timing information derived from rate, including an abrupt increase in heart rate, detection of a rate which exceeds an arbitrary minimum, and obeervation of a atable RR interval during the tachycardia. Such algorithmo lack specificity for VT and VF recognition, however $[5,6]$.
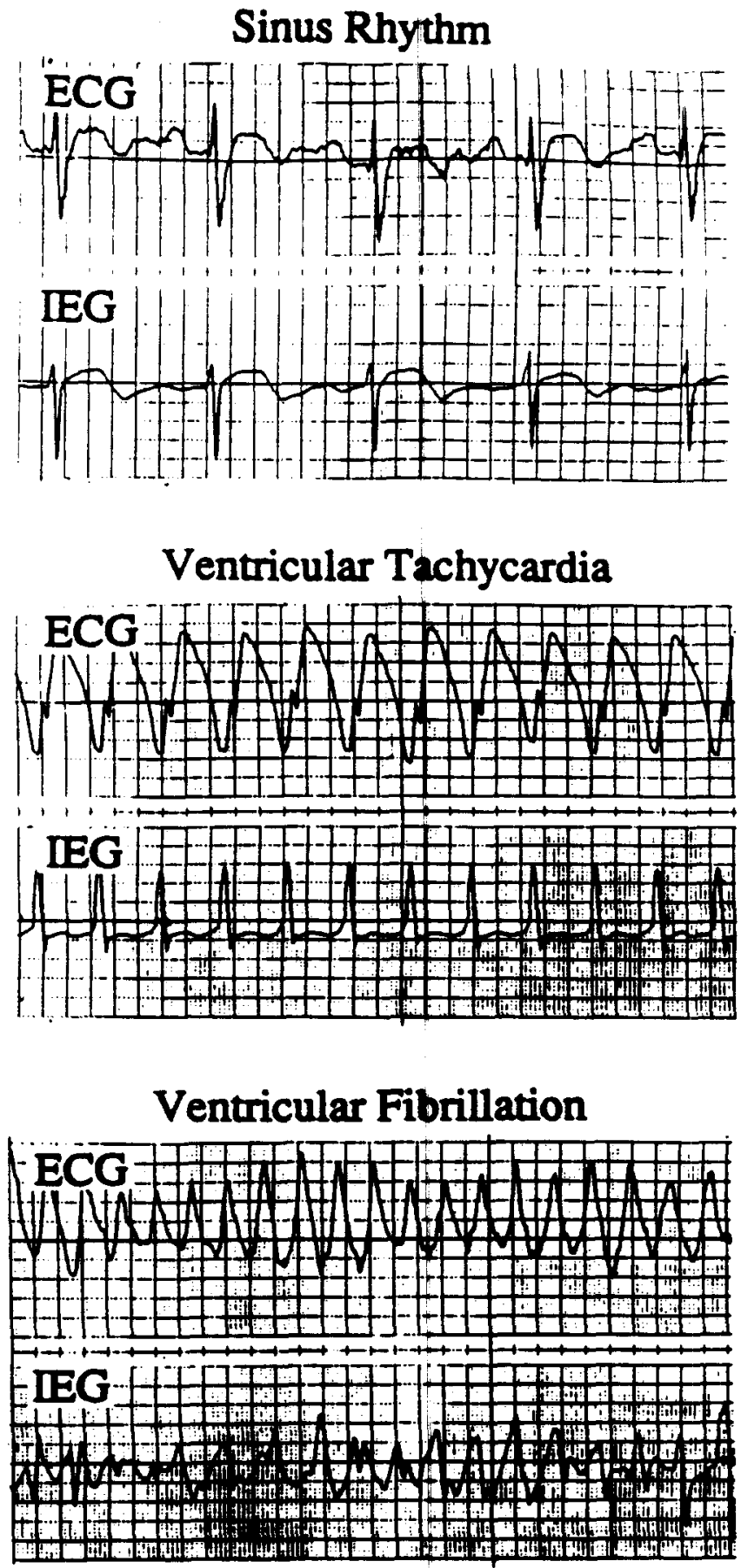

Figure 1. Exemples of the surface electrocandiographic (ECG) and distal bipolar intraventriceler electrograms (IEG) of patieat No. 11 during siaus rhythm (SR), monomorphic ventricular tachycardie (VT), and ventricalar fibrillation (VF). The difference in mean CC from CWA of SR (.959) differentiated it from $V T(.040)$ and $V F(.162)$. The verience of $V T$ $(.177)^{2}$ differenticted it from VF (.199) 

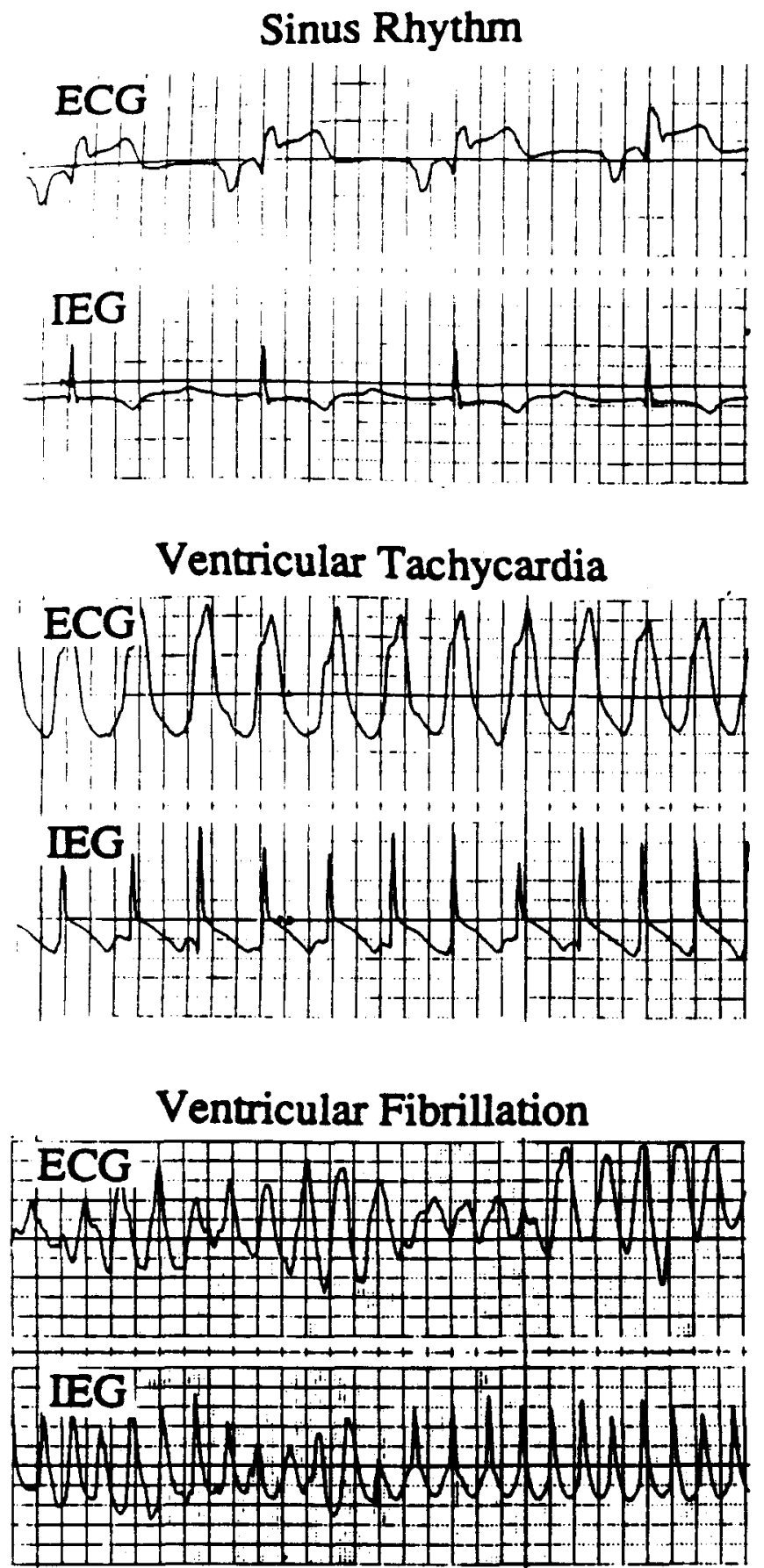

Figure 2. Examples of the electrogrems of petient No. 10 during $S R, V T$ and $V F$. The difference in mean CC from $C W A$ of $S R$ (.977) differentiated it from $V T$ (.960) and VF (.881). The verience of VT $(.018)^{2}$ and VF (.092) were similar, however. Discrimine. tion between these two tachyarrhythmias wes not poesible using this criterion. The abbreviations are similar to those of Figure 1.
Both time-domain and frequency-domain analysis have been propoeed for VT and VF detection $[1,2,3,4,7]$. Only one method-probability density function-has been incoporporated into an implantable device for VF detection. However, it has had limited applicability for patients who experience both recurrent VT and VF. In many cases, the activity peak of VT determined by this method of amplitude distribution analysis may occur at amplitude zero, resulting in failure to detect VT [8].

Previous studies have demonstrated the efficacy of correlation waveform analysis in detecting VT when compared to other proposed time- and frequencydomain analyses of individual bipolar intraventricular electrograms. The results of the present study suggest a similar time-domain analysis of intraventricular bipolar electrograms may also be useful in diseriminating VF from SR as well as from more organized and more stable monomorphic VT. Although the values of CWA of VT and VF may be similar, this study has demonstrated that the variances of the correlation coefficient of VT are significantly different from those of VF. The magnitude of the variance in VT and VF appears to be useful in distinguishing these two ventricular tachyarrhythmias.

CWA has been utilized in previous studies of other arrhythmias as a "gold standard" against which other methods of time-domain analysis have been compared. Although CWA has potential advantages of being independent of eleetrogram amplitude and baceline fluctuations, its incorporation in currently available, implantable microprocessors is impractical because of its computational demands. Further work will be necessary to determine whether other, less computationally demanding time-domain methods [9] will be similarly effective in the detection and discrimination of VT and VF.

\section{Acknowledgments}

Thi research was supported in part by National Seience Foundation Grant BCS-890942.

\section{References}

[1] DiCarlo LA, Throne RD, Jenkins JJ. A timedomain analyois of intracardiac electrograms for arrhythmia detection. PACE 1991; 14:329-336.

[2] Lin D, DiCarlo LA, Jenkins JJ. Identification of ventricular electrograms: Analysis of time and frequency domain patterns. PACE 1988; 11:1592-1606. 
[3] Throne RD, DiCarlo LA, Jenkins, Winston SA. Paroxysmal bundle branch block of supraventricular origin: A possible source of misdiagnosis in detecting ventricular tachycardia using timedomain analysis of intraventricular electrograms. PACE 1990; 13:453-468.

[4] Throne RD, Jenkins JJ, Winston SA, Finelli CJ, DiCarlo LA. Discrimination of retrograde from anterograde atrial activation using intracardiac electrogram waveform analysis. PACE 1989; 12:1622-1630.

[5] Charos GS, Haffajee CI, Gold RL et al.: A theoretically and practically more effective method for interruption of ventricular tachycardia: Selfadapting autodecremental overdrive pacing. Circ 1986; 73:309-315.

[6] Olson WH, Bardy GH, Mehra R et al.:
Tachyarrhythmia detection algorithm for an implantable cardioverter and defibrillator. Circ 1986;74II-110.

[7] Davies DW, Wainwright RJ, Tooley MA et al.: Endocardial electrogram analysis for the automatic recognition of ventricular tachycardia. Circ 1985; 72:III-474.

[8] Mirowski M, Mower MM, Reid PR et al.: The automatic implantable defibrillator, new modality for treatment of life-threatening ventricular arrhythmias. PACE 1982; 5:384-401.

[9] Throne RD, Jenkins JJ, DiCarlo LA. A comparison of four new time-domain techniques for discriminating monomorphic ventricular tachycardia from sinus rhythm using ventricular waveform morphology. IEEE Transactions on Biomed. Engin. 1991; 38:561-570. 